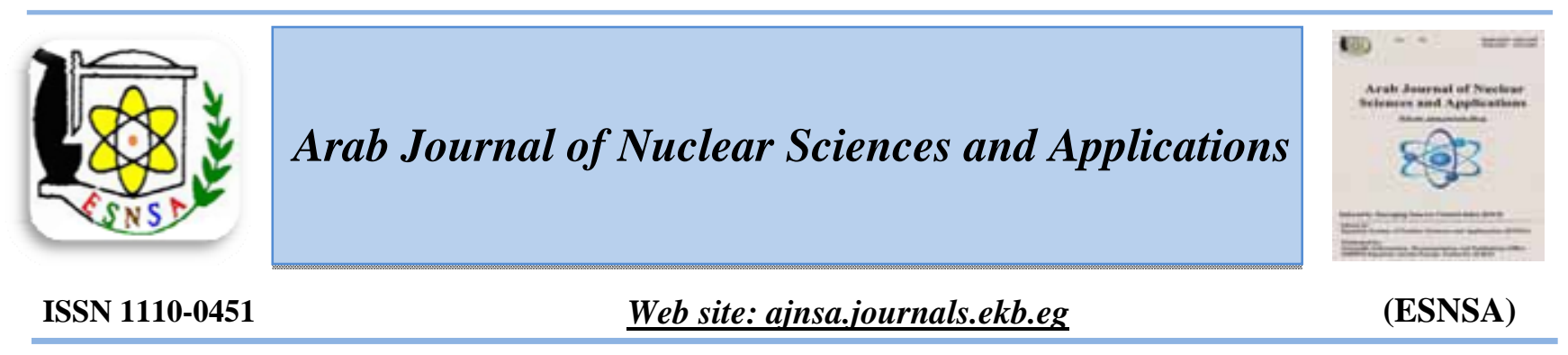

\title{
Effect of the Number Shots of Laser on Structural Transformations and Optical Properties of ZnS Nanoparticles Thin Films
}

\author{
A.Z. Mohammed ${ }^{1}$, N.J. Mohammed ${ }^{2 *}$, I.K. Khudhair ${ }^{2}$ \\ (1) Physics Department, Faculty of Science, Zagazig University, Zagazig, Egypt \\ ${ }^{(2)}$ Physics Department, Faculty of Science, Al-Mustansiriyah University, Iraq
}

Received $30^{\text {th }}$ Jul 2017 Effects of the number of shots of laser and annealing temperature on the structural, morphological, and Accepted $16^{\text {th }}$ Aug 2018 optical properties of zinc sulfide nanoparticles (ZnS NPs) thin films, prepared by pulsed laser deposition technique (PLD), were investigated. XRD results show that ZnS NPs exhibit a hexagonal phase at 623k. Transformation in the shape of nanoparticles to nanoflowers and nanorods appeared with increasing number of shots of laser at the same annealing temperature (623K) was observed from scanning electron microscopy (SEM) images. The optical properties were studied from all transmittance data. The experimental results show that the as-deposited ZnS NPs film exhibit a cubic structure and the crystallinity increased in the annealed films. It is also found that the grain size of the as grown samples at $300 \mathrm{~K}$, rises linearly from 9 to $11 \mathrm{~nm}$ with increasing the number of shots from 1000 to 3000 and rapidly from 10 to $18 \mathrm{~nm}$ after annealing at $623 \mathrm{~K}$. Additionally, the increase of the number of shots from 1000 to 3000 leads to a decrease in the energy gap values and increases their values after heat treatment keeping their behavior decreasing as the number of shots of laser increases. Also, photoluminescence (PL) measurements explained quenching its value after annealing temperature.

Keywords: ZnS, Pulsed laser deposition, Nanoparticles, Nano leaves, Nanoflowers, Nanorods, Photoluminescence (PL), Annealing temperature

\section{Introduction}

$\mathrm{ZnS}$ is a II-VI compound semiconductor. It is widely applied in the optoelectronic applications consisting of light emitting diodes with short wavelength. The structure and properties of $\mathrm{ZnS}$ films vary according to the deposition technique. Many growth techniques have been reported to prepare $\mathrm{ZnS}$ thin films, such as sputtering [1], Pulsed-laser deposition [2], metal organic chemical vapor deposition [3], electron beam evaporation [4], photochemical deposition [5], and chemical bath deposition [6]. Among these methods, electron beam evaporation is the most interesting. The advantages of electron beam evaporation are stability, reproducibility; high deposition rate, moreover, the compositions of the films are controllable. $\mathrm{ZnS}$ is one of the first semiconductors discovered [7], and is also an important semiconductor material with direct wide band gaps for cubic and hexagonal phases of 3.72 and 3.77 $\mathrm{eV}$, respectively [8]. $\mathrm{ZnS}$ has a high absorption coefficient in the visible range of the optical spectrum and reasonably good electrical properties [9]. In recent years, $\mathrm{ZnS}$ thin films have been grown by a variety of deposition techniques, such as chemical bath deposition [10], evaporation [11], and solvo thermal method [12]. Chemical bath deposition is a promising technique because of its low cost arbitrary substrate shapes, simplicity, and capability of large area preparation. There are many reports of successful fabrication of $\mathrm{ZnS}$ based hetero-junction solar cells by utilizing the chemical bath deposition method, such as with CIGS used for the n-type emitter layer [13]. The much pulsed laser deposition experiments were carried out in the 1960s, however it was first

Corresponding author: N.J. Mohammed

DOI: $10.21608 / a j n s a .2018 .12602$

(C) Scientific Information, Documentation and Publishing Office (SIDPO)-EAEA 
popularized during the 1980 s by the work of Inamet et al. [14], who deposited high temperature super conducting films with a complex stochiome $\mathrm{ZnS}$ is potentially important material to be used as an antireflection coating for hetero-junction solar cells [15]. In this research A novel method was used involving changing the number of shots of laser to produce $\mathrm{ZnS} \mathrm{NPs}$, nanoflowers and nanorods at the same heat treatment temperature $(623 \mathrm{~K})$ without inlet gas to the vacuum chamber.

\section{Experimental}

The ceramic $\mathrm{ZnS}$ target used is a pellet with dimensions $(2 \mathrm{~cm} \times 0.1 \mathrm{~cm})$, density $3.66 \mathrm{~g} / \mathrm{cm}^{3}$ and purity $99.99 \%$, supplied by the Kurt J. Lesker Company. It is produced by pressing squeeze at 13 ton, and sintering $\mathrm{ZnS}$ powder under vacuum at $1125 \mathrm{~K}$. It is a suitable target for PLD since it is dense and flat, enabling uniform energy transfer to its surface and the absence of voids keeps large particles being ejected from the surface. ZnS NPs films were prepared by pulsed laser deposition system of $\mathrm{ZnS}$ pellet fixed to a target holder that located at $2 \mathrm{~cm}$ and parallel to the substrate surface. The PLD was carried out by using a Q- switched Nd: YAG laser with wave length (1064 $\mathrm{nm})$, number of shots of laser $(1000,2000,3000)$, laser flounce $\left(16.98 \mathrm{~J} / \mathrm{cm}^{2}\right)$ and spot $(\mathrm{d}=3 \mathrm{~mm})$ at an angle $0 \mathrm{f} 45^{\circ}$. The repetition rate of the laser beam was $5 \mathrm{~Hz}$. The target and substrate were rotated at 10 and $6 \mathrm{rpm}$ respectively, by using DC motor to avoid drilling effect. The chamber of substrate and target holder evacuated to a pressure about $10^{-5}$ mbar. The preparation temperatures of samples were as grown temperature $(300 \mathrm{~K})$ and annealing temperature $(623 \mathrm{~K})$. In a typical case $\left(16.98 \mathrm{~J} / \mathrm{cm}^{2}\right.$, $1064 \mathrm{~nm}$ ) the thickness created after deposition by 1500 pulses was found to be $50 \mathrm{~nm}$.

\section{Results \& Discussion}

The structure of $\mathrm{ZnS}$ target was investigated using $\mathrm{X}$-ray diffraction, as shown in Figure (1), and it was found that $\mathrm{ZnS}$ exhibits hexagonal phase. $\mathrm{ZnS}$ NPs thin films were prepared in an evacuated chamber to a base pressure of $\left(10^{-5} \mathrm{mbar}\right)$. All three films, prepared by different number of shots of laser $(1000,2000$, and 3000) at room temperature $(300 \mathrm{~K})$, were annealed at $623 \mathrm{~K}$. The crystal structure of the films was examined by Xray diffraction (XRD) as shown in Figure (2).

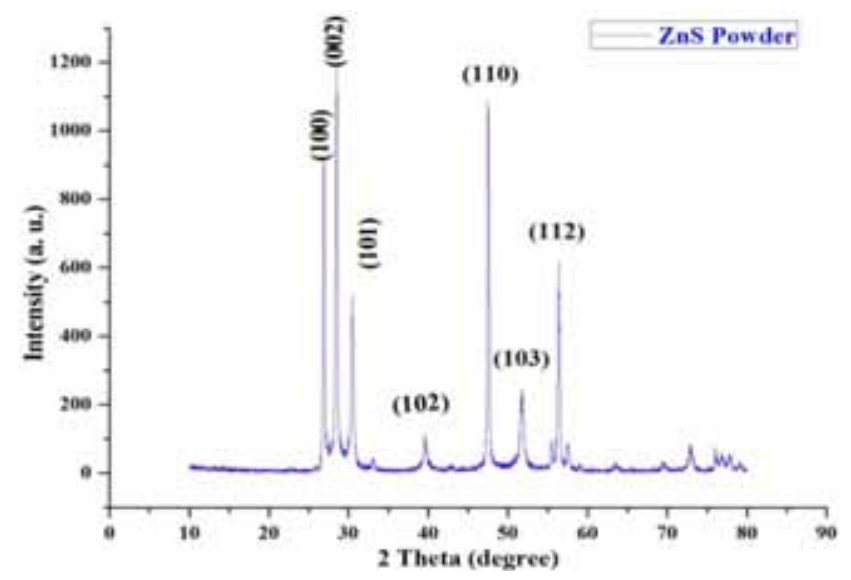

Figure (1): X-ray diffraction patterns for ZnSta target

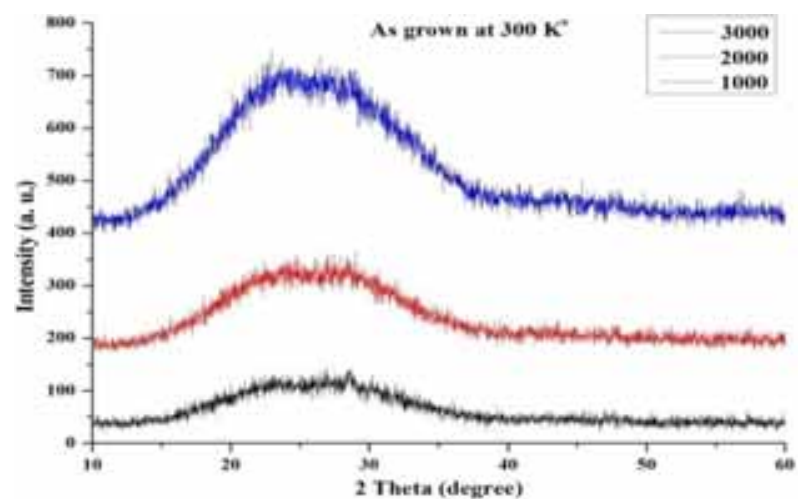

(a)

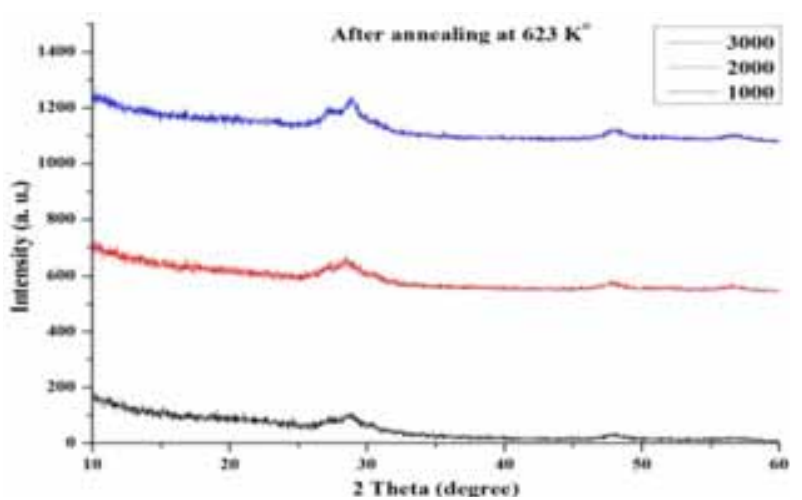

(b)

Figure (2): X-ray diffraction patterns for ZnS NPs films at a) as grown $300 \mathrm{~K}$, b) annealed at $623 \mathrm{~K}$ 
The average crystallite size $(D)$ of $\mathrm{ZnS}$ NPs was estimated by the standard Scherrer formula [16]

$D=\frac{k \lambda}{\beta \cos \theta}$

Where $k$ is constant $(0.89<k>1), \beta$ is the Full Width at Half Maximum (FWHM) of the diffraction peak, $\lambda$ is the wavelength of the $\mathrm{X}$-ray and $\theta$ is diffraction angle. At different no. shots of laser $(1000,2000,3000)$, it can be observed that the crystallite size of the samples increased after annealing from $(9,9.5,11 \mathrm{~nm})$ at $(300 \mathrm{~K})$ to $(10,11$, $18 \mathrm{~nm}$ ) at 623K.as shown in Figure (3).
Also, it can be noticed that the differences in the crystallite size at $300 \mathrm{~K}$ and at annealed temperature $(623 \mathrm{~K})$ increased with increasing no. of pulses as shown in Fig (4).

Further investigations on the characteristics of nanosized structures of the deposited materials were achieved using atomic force microscopy. The AFM image of the ZnS NPs thin films deposited by different no. shots $(1000,2000,3000)$ at $300 \mathrm{~K}$ are shown in Figure(5).

The AFM image of the ZnS NPs thin films deposited by different number of shots (1000, 2000 , and 3000 ) after annealed at $623 \mathrm{~K}$ is illustrated in Figure(6).

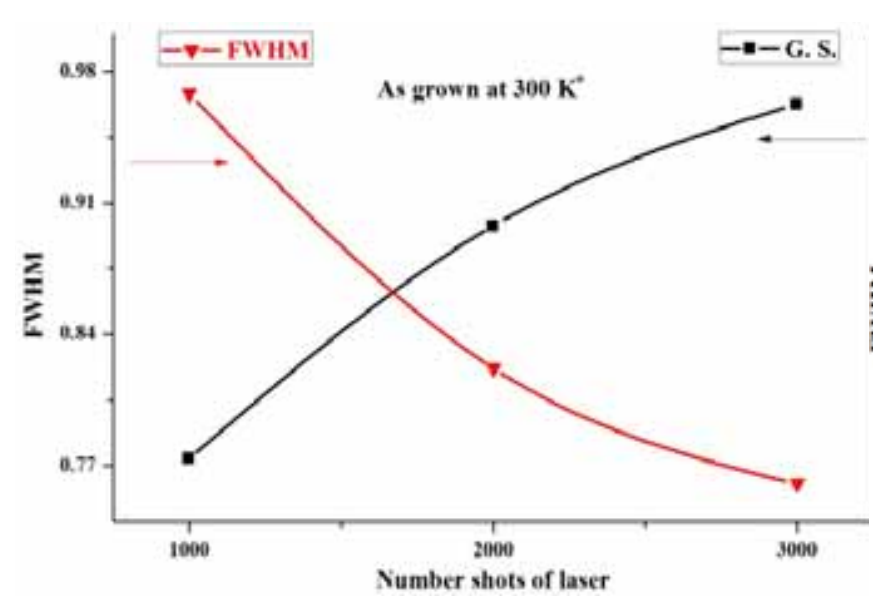

(a)

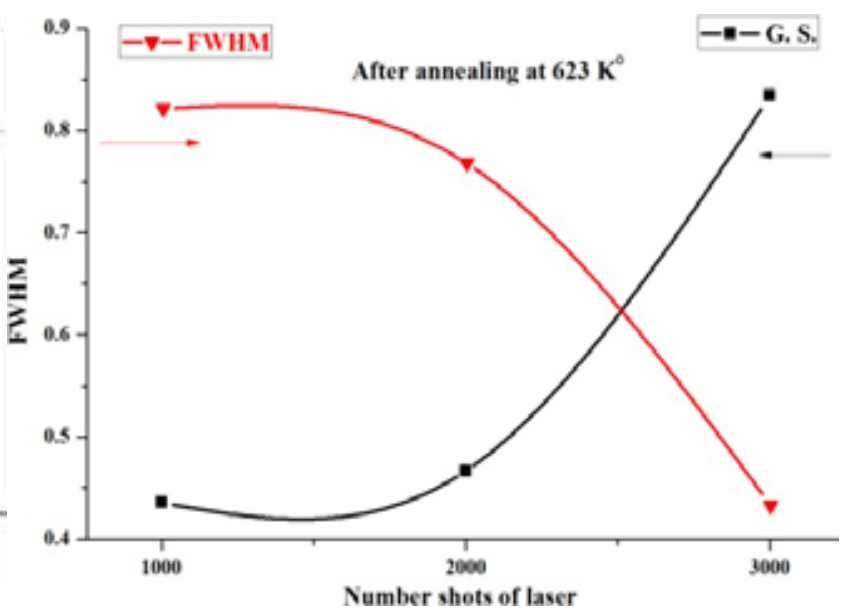

(b)

Figure(3): The crystallite size verse FWHM of ZnS NPs films for different no. shots (1000, 2000, 3000) at: a) as grown $300 \mathrm{~K}, \mathrm{~b})$ annealed at $623 \mathrm{~K}$

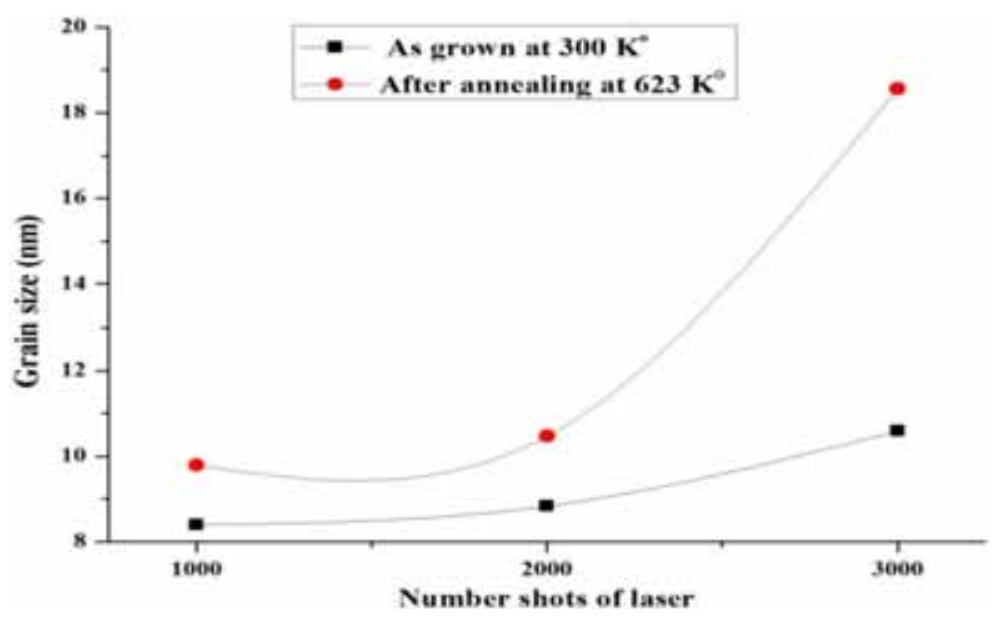

Figure (4): The differences in the crystallite size at $300 \mathrm{~K}$ and at annealing temperature (623k) 

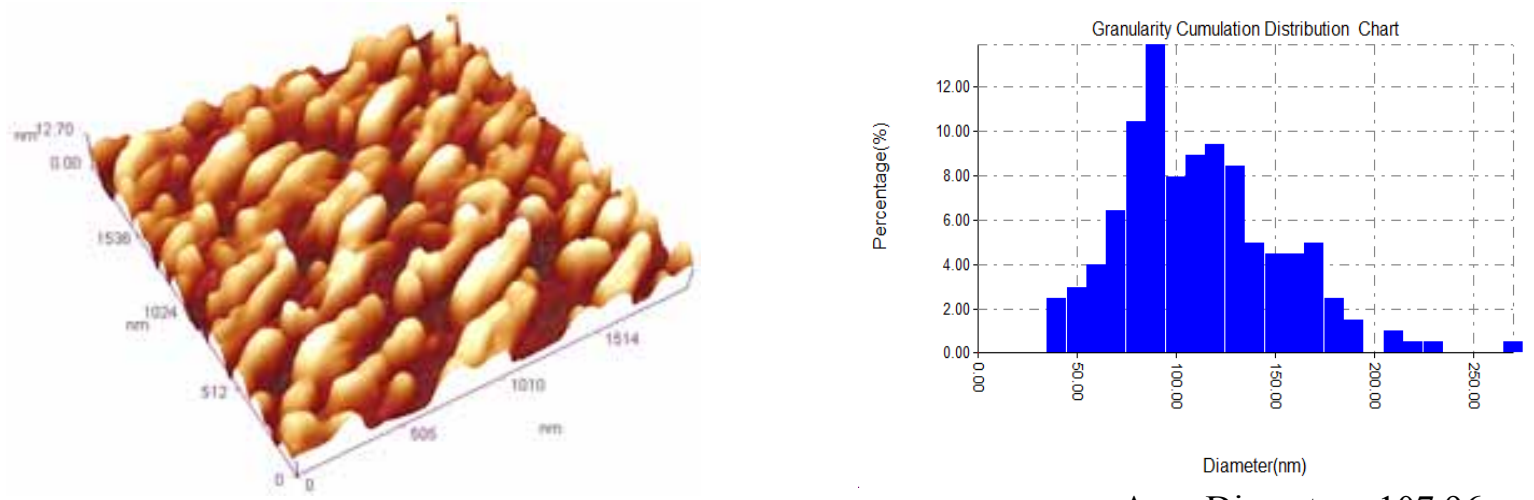

Avg. Diameter : 107.96 nm
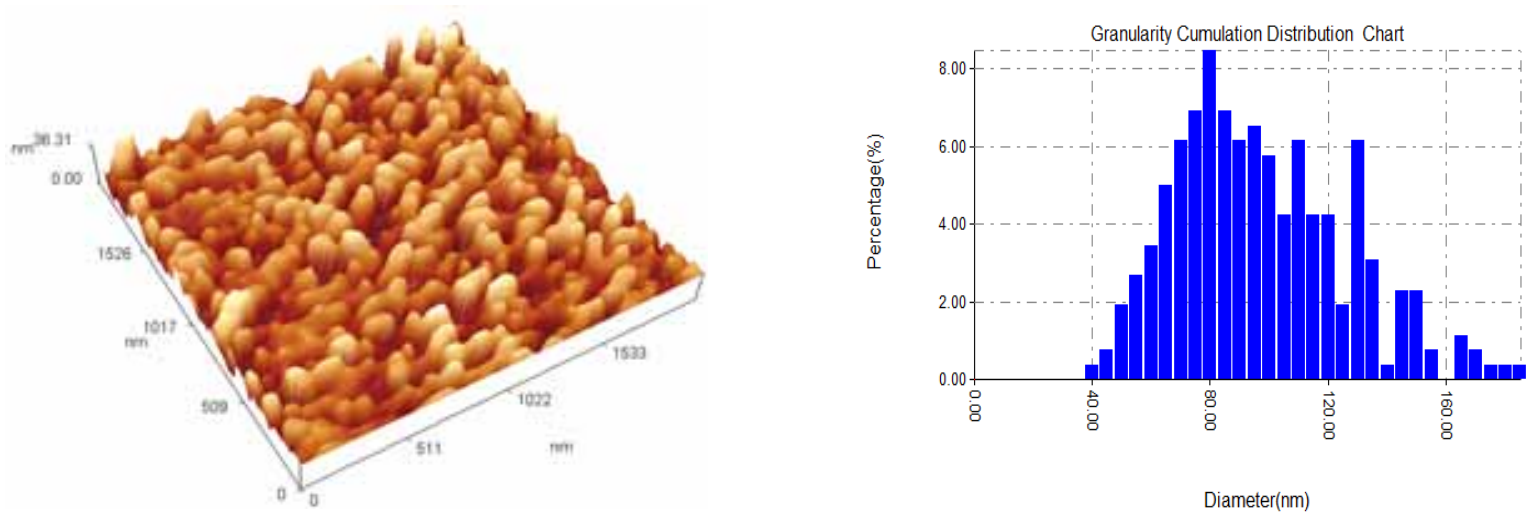

Avg. Diameter: $94.93 \mathrm{~nm}$
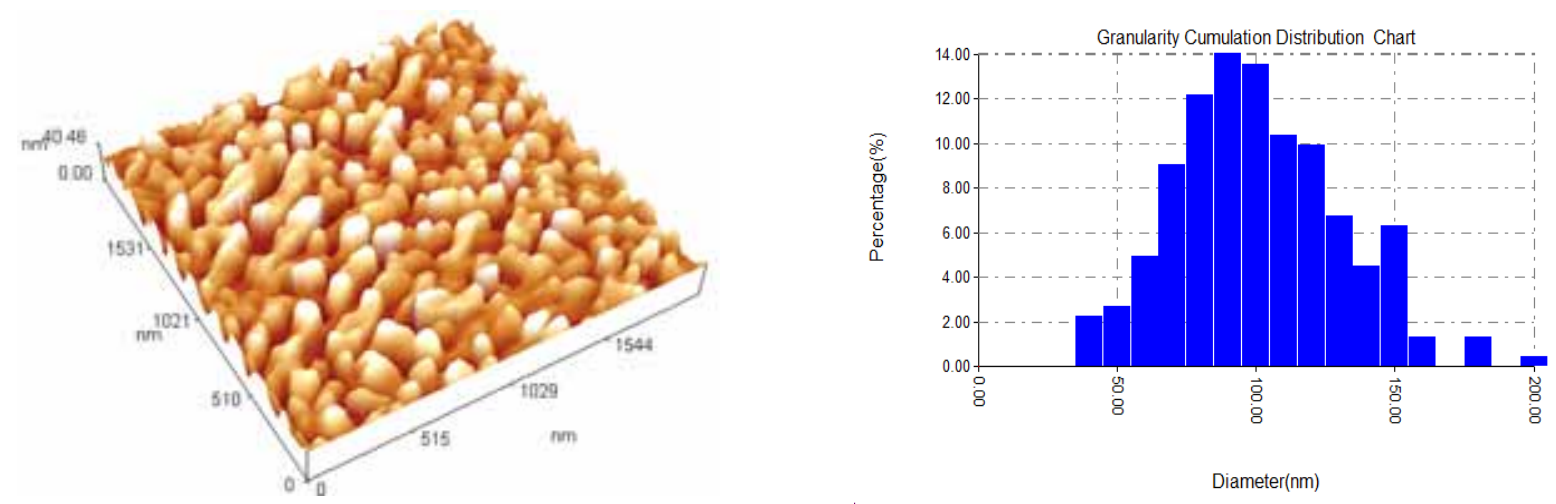

Avg Diameter 95.95nm

Figure (5): AFM of ZnS NPs thin films deposited by different shots (1000, 2000, 3000) with average grain size (107.96, 94.93 and $95.95 \mathrm{~nm}$ ) respectively at $300 \mathrm{k}$ before annealing 

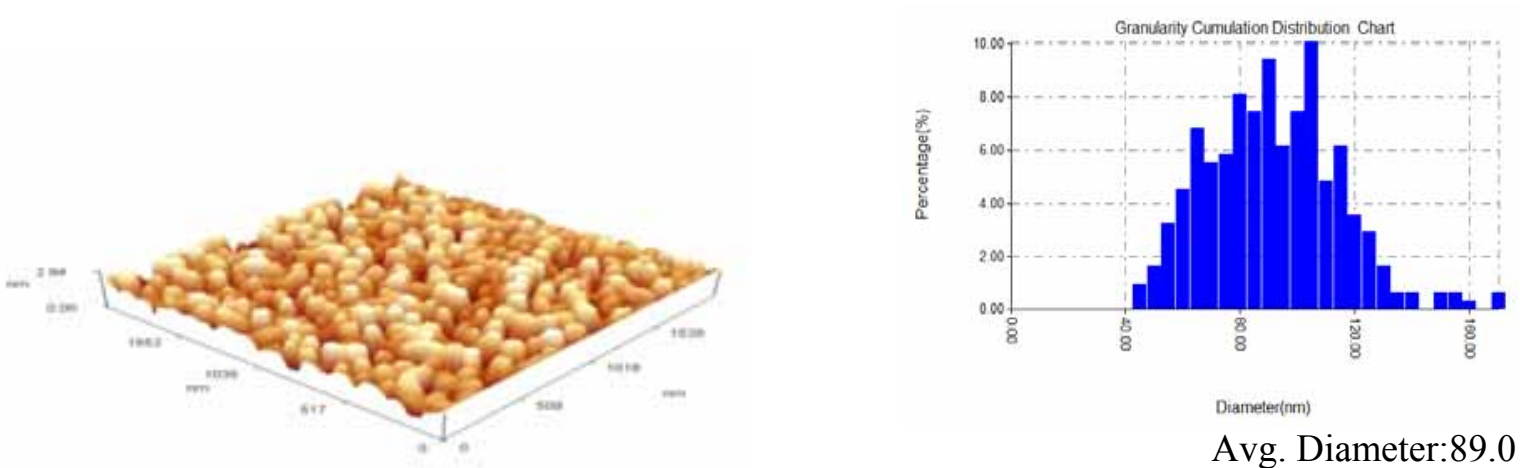

Avg. Diameter:89.01nm
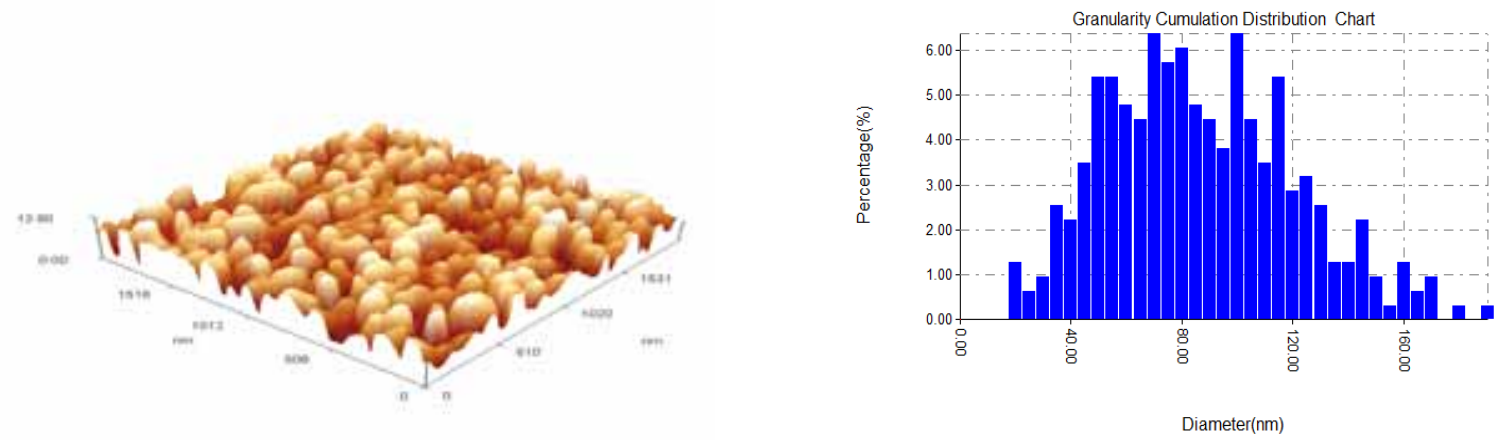

Avg. Diameter:84.55nm
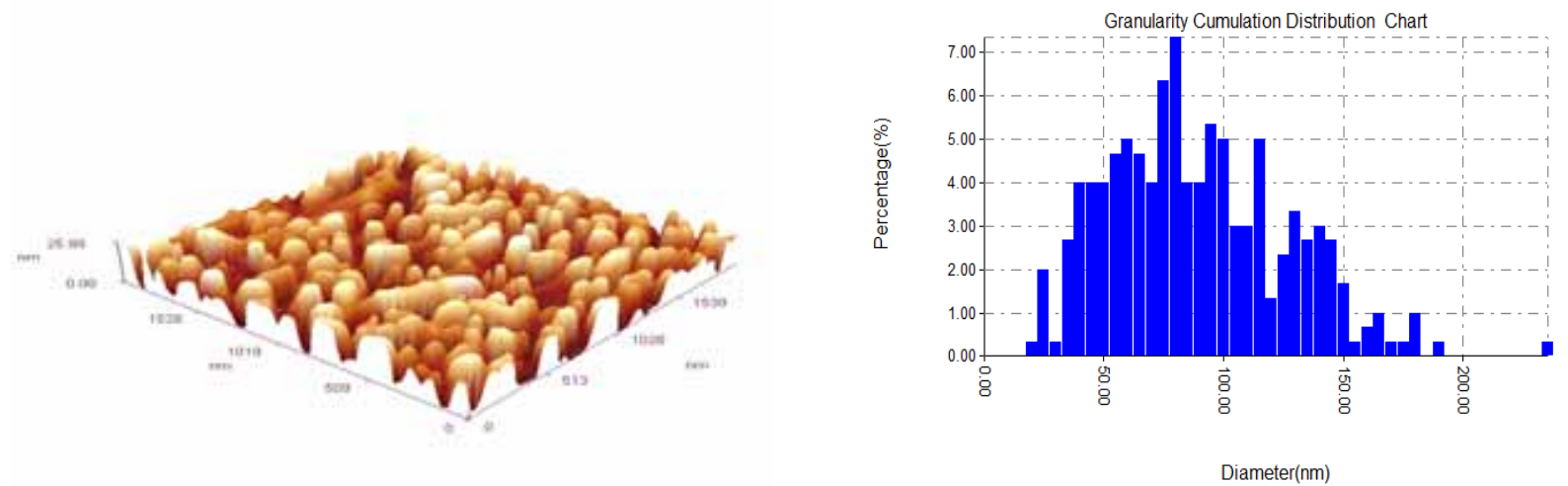

Avg. Diameter:86.32nm

Figure (6): AFM of ZnS NPs thin films deposited by different shots (1000, 2000, 3000) with average grain size (89.01, 84.55 and $86.32 \mathrm{~nm}$ ) respectively after annealing at $623 \mathrm{~K}$

The average size of $\mathrm{ZnS}$ NPs was about $99 \mathrm{~nm}$ at $300 \mathrm{~K}$ and the average size decreased to $86 \mathrm{~nm}$ after annealing samples at $623 \mathrm{~K}$. The root mean square (rms) surface roughness at $300 \mathrm{~K}$ for the prepared films with different no. shots $(1000$, 2000, and 3000) are 3.61, 7.78 and $8.36 \mathrm{~nm}$ respectively. After annealing the samples at $623 \mathrm{~K}$ the (rms) the surface roughness decreased for each sample and increased with increasing number of shots of laser for all samples. Table (1) shows the surface roughness of $\mathrm{ZnS}$ NPs thin film deposited on glass substrate. Increasing number of shots leads to an increased roughness of the surface and increasing the temperature could reduce it. The decrease in (rms) surface roughness may be due to the decrease in the number of point defects which means a decrease of dislocations density $(\delta)$ in the films as shown in Table (1).

The SEM images are presented in Figure(7) as a function of the number of shots of laser and annealing temperature. Figure7(a) shows the SEM image of a synthesized sample at $300 \mathrm{~K}$ for 1000 shots, the nanoparticles transformed from circular to nanoflowers-like particles as a result of the 
accumulation of nanoleaves generated by nanoparticles from the effect of heat treatment at $623 \mathrm{~K}$. We noticed that when the number of shots 2000, the nanoparticles take the form of nanorods with a diameter ranging from 50 to 150 nanometers with an increase in the number of shots to 3000 after annealing at $623 \mathrm{~K}$ as illustrated by
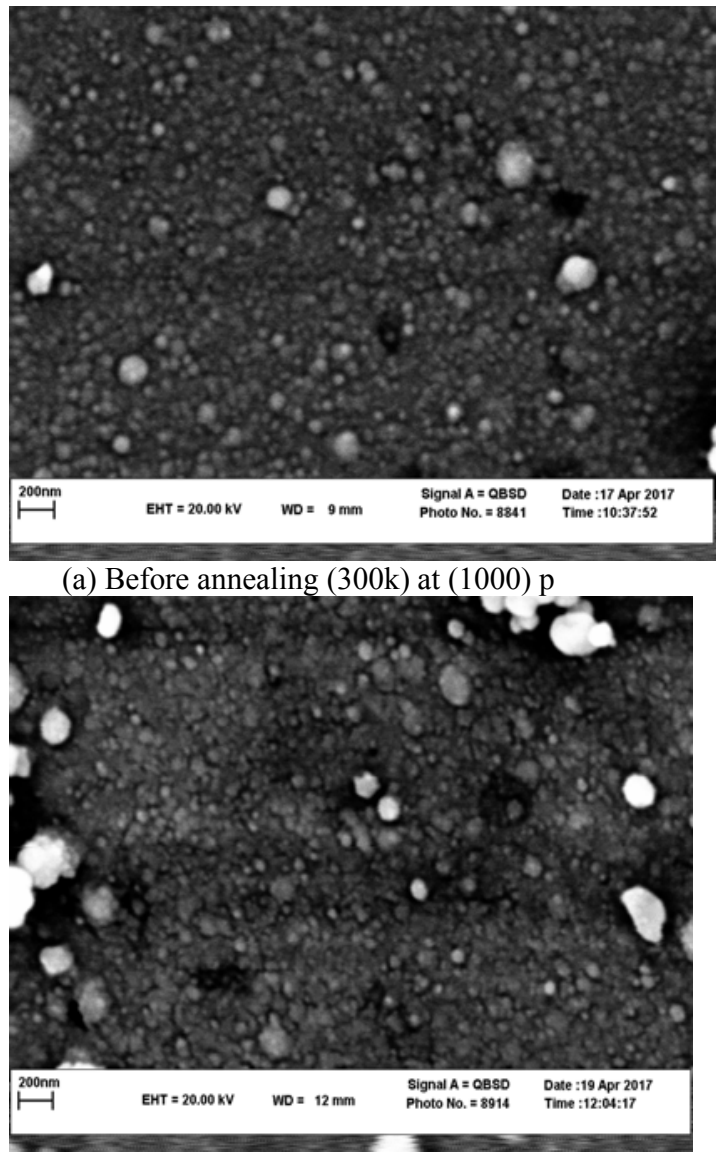

(a) Before annealing (300k) at (2000) p

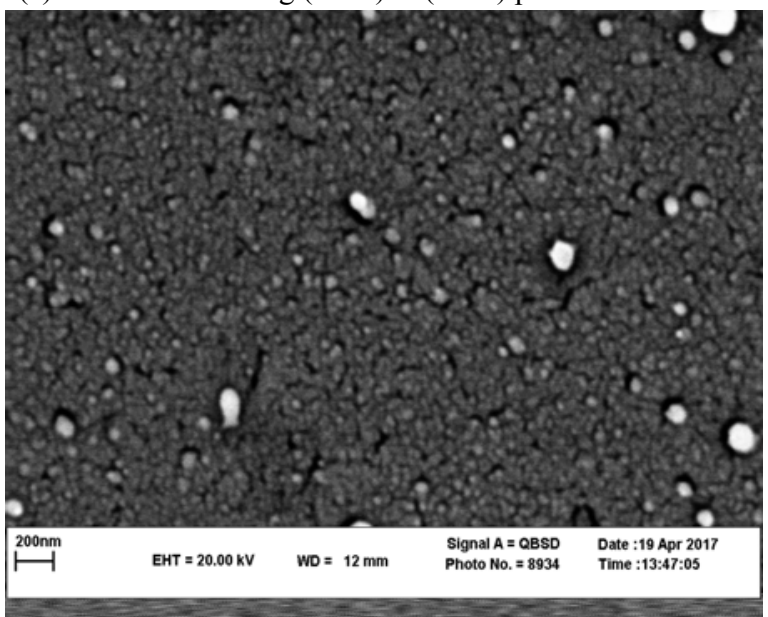

(c) Before annealing (300k) at (3000) p
Figure(7) (b) and (c) respectively, which are confirmed by XRD results. Annealing of particles at various temperatures could result in the agglomeration of particles as shown in Figure(4). At higher annealing temperatures, the nanoparticles are merging with each other forming a neck between the two particles.
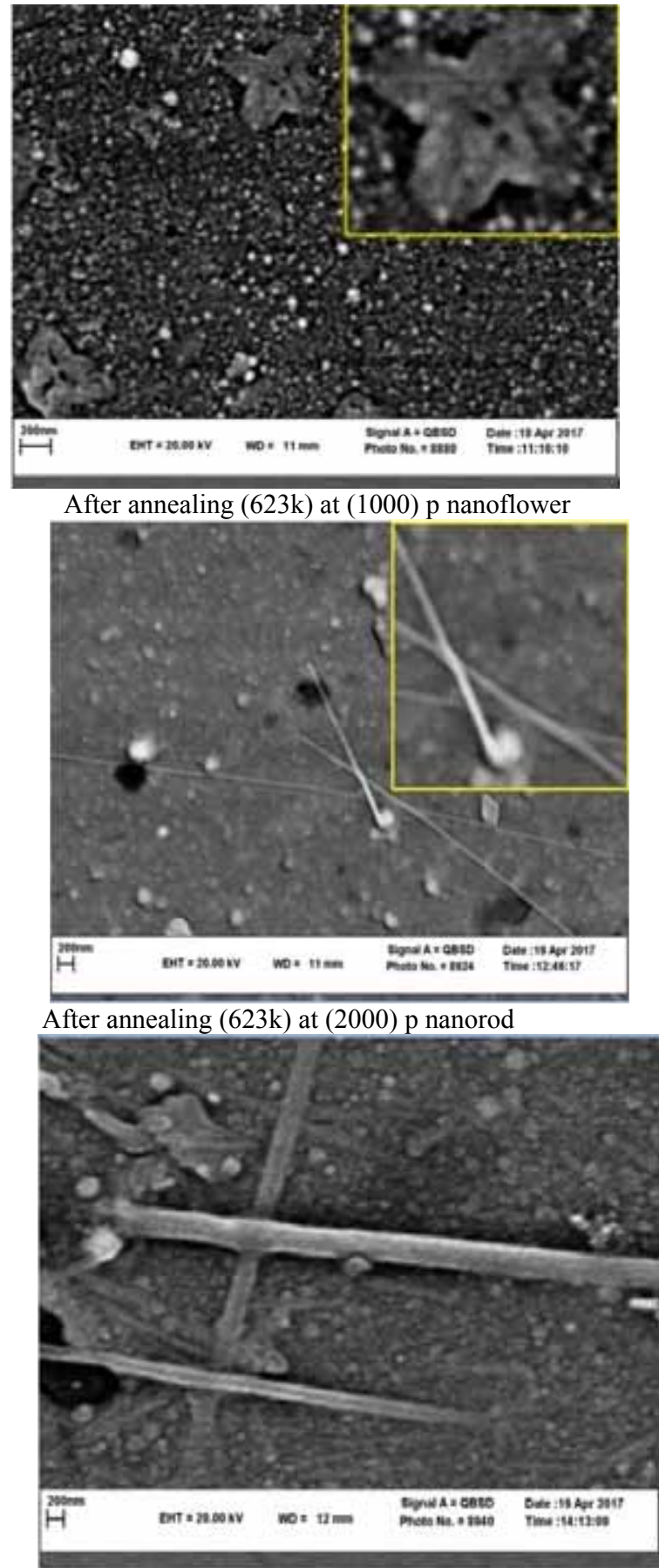

After annealing (623k) at (3000) p nanorod

Figure (7): SEM images of ZnS NPs thin films, the particles are shown for 1000 shots (a); and for 2000 shots (b); and for 3000 shots (c) at (300K) and annealing (623K) 
Table (1): surface roughness of ZnS NPs films prepared at temperature (300k) and (623k)

\begin{tabular}{cccc}
\hline $\begin{array}{c}\text { Preparation } \\
\text { temperature (K) }\end{array}$ & No. of laser shots & $\begin{array}{c}\text { Root mean square surface } \\
\text { roughness }(\mathrm{nm})\end{array}$ & $\begin{array}{c}\text { Dislocations } \\
\text { density }(\delta)(\mathrm{nm})^{-2}\end{array}$ \\
\hline $\begin{array}{c}\text { As grown at } \\
(300 \mathrm{~K})\end{array}$ & 1000 & 3.61 & 0.0142 \\
& 2000 & 7.78 & 0.0103 \\
After annealing at & 3000 & 8.36 & 0.0089 \\
$(623 \mathrm{~K})$ & 1000 & 0.674 & 0.0091 \\
& 2000 & 3.17 & 0.0059 \\
& 3000 & 7.47 & 0.0029 \\
\hline
\end{tabular}

Interference peaks in the Figure (8). explained the uniform of the $\mathrm{ZnS}$ NPs films at $300 \mathrm{~K}$ and the transmittance was found to increase with increasing number of shots of laser. After annealing at $623 \mathrm{~K}$.

The direct energy band gap $\left(E_{g}\right)$ was estimated from transmittance data as a function of wavelength using the relation [17]; $\alpha h v=$ $\left(h v-E_{g}\right)^{\frac{1}{2}}$, where $h v$ is the photon energy $(\mathrm{eV})$. The energy gap $\left(\mathrm{E}_{\mathrm{g}}\right)$ of $\mathrm{ZnS}$ NPs films prepared at $300 \mathrm{~K}$ and $623 \mathrm{~K}$ calculated by extrapolating the linear part of $(\alpha h v)^{2}$ vs. $(h v)$ curves to $(\alpha h v)^{2}=0$ as shown in the Figure (9).

The band gap values estimated in this work for annealed ZnS NPs films at 623K (3.569, 3.488, $3.377 \mathrm{eV}$ ) are found to be higher than that prepared at $300 \mathrm{~K}(3.557,3.449,3.319 \mathrm{eV})$, which may be linked with the structural changes causing quantum confinement effects in the ZnS NPs films as shown in Figure(10).
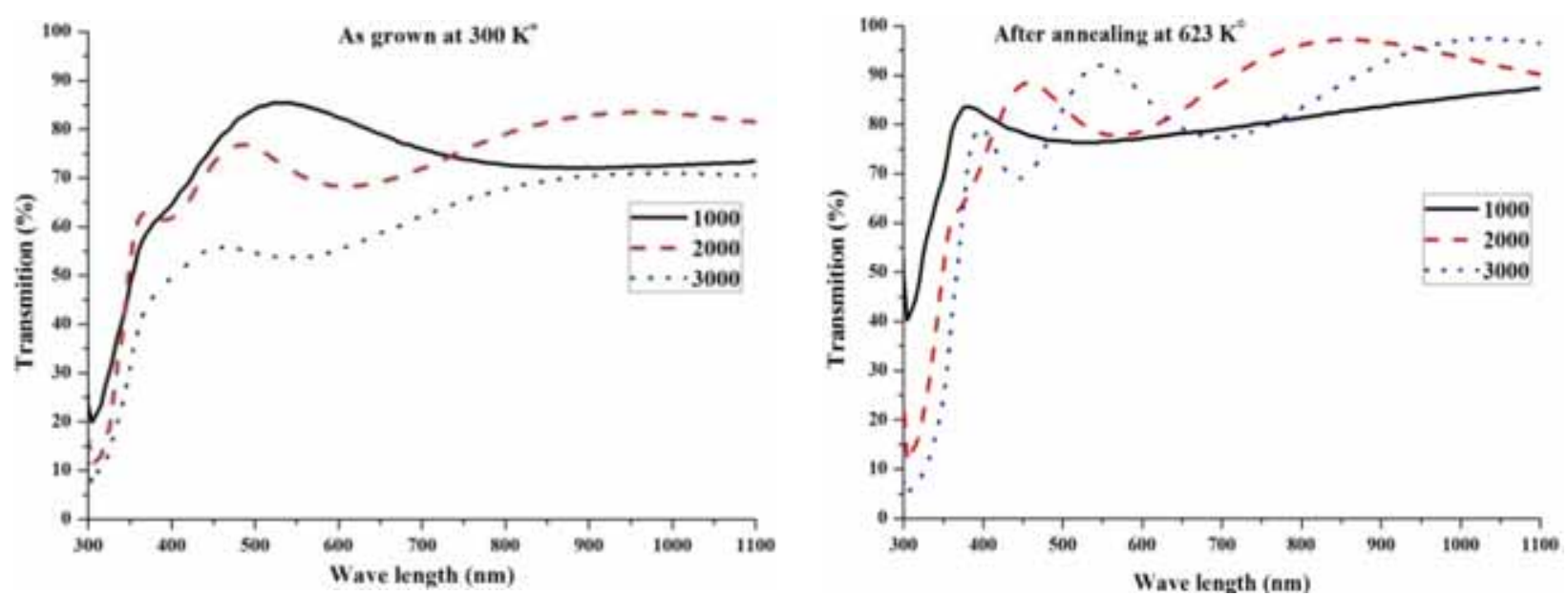

Figure (8): Optical transmission of the ZnS NPs films prepared at $300 \mathrm{~K}$ and annealed temperature (623K)
Also, the decrease in the values of $E_{g}$ by increasing number of laser shots $(1000,2000$, $3000)$ is due to the increase in the crystal size of $\mathrm{ZnS}$ NPs. Photoluminescence (PL) spectrum of $\mathrm{ZnS}$ NPs films, which was prepared with a number of laser shots $(1000)$ and flounce $\left(16.98 \mathrm{~J} / \mathrm{cm}^{2}\right)$ at $(300 \mathrm{~K})$ and annealed at $623 \mathrm{~K}$, was measured in the region from 350 to $700 \mathrm{~nm}$ with excitation wave length $(350 \mathrm{~nm})$. Figure(11) shows ZnS NPs films grown $(300 \mathrm{~K})$ and there are two evident peaks that give the ultraviolet (UL) and blue luminescence (BL) emission bands at 396 and $437 \mathrm{~nm}$, respectively.

After annealing at $623 \mathrm{~K}$, these bands were quenched and shifted to 397 and $439 \mathrm{~nm}$ respectively as shown in Table (2).

This emission bands originated from zinc oxide and sulfur vacancies. The quenching of (PL) was attributed to the oxidation of sulfur into sulfate and the removal of lattice defects by the annealing process. 


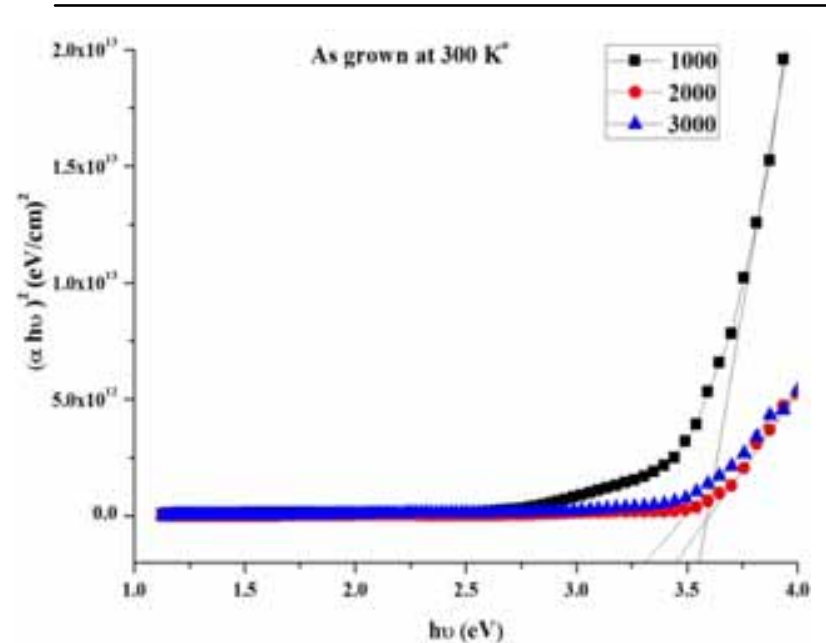

(a)

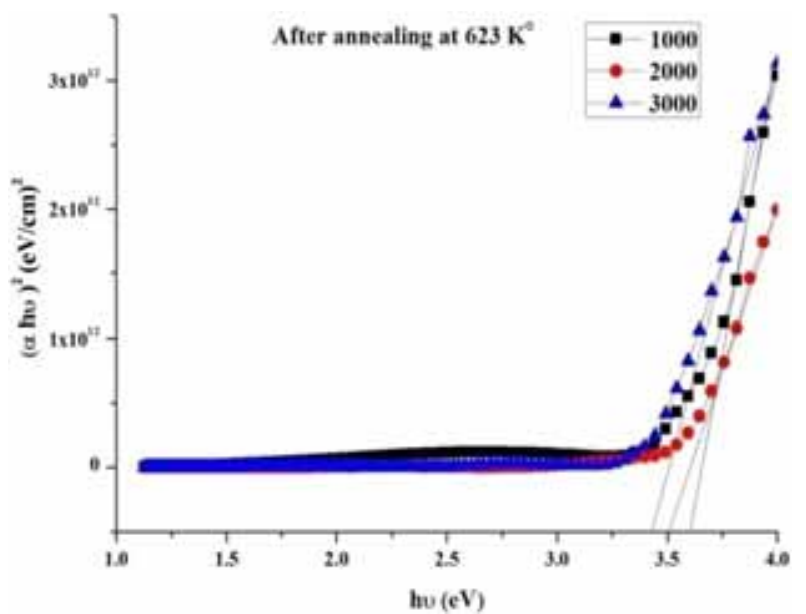

(b)

Figure (9): The energy gap ( $\left.E_{g}\right)$ of ZnS NPs films prepared at (a) $300 \mathrm{~K}$ and (b) 623K

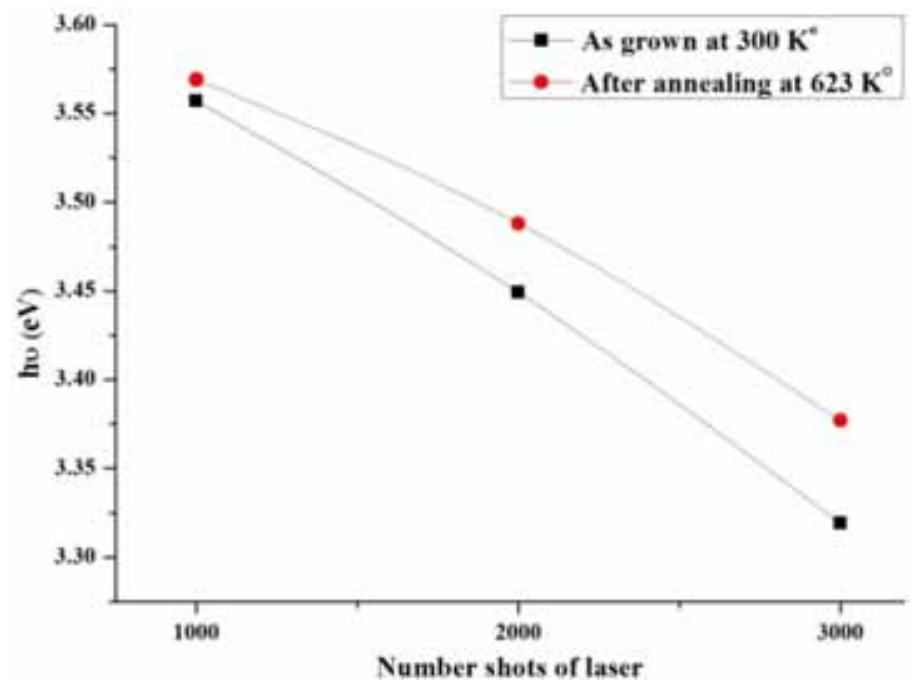

Figure (10): The $E_{g}$ of ZnS NPs films prepared at temperature $300 \mathrm{~K}$ and $623 \mathrm{~K}$

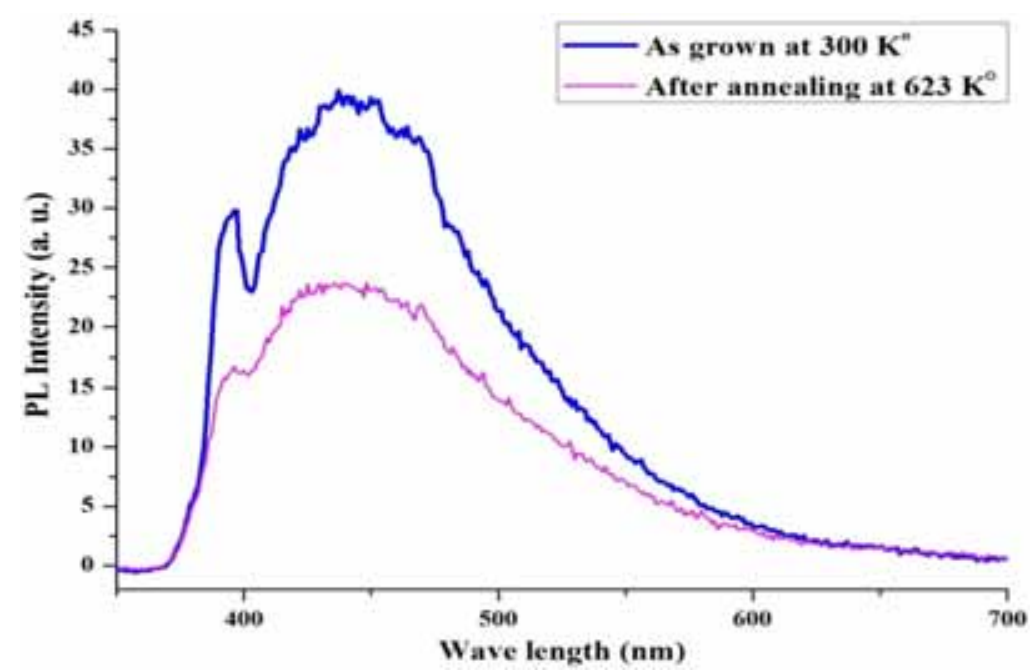

Figure (11): Room temperature Photoluminescence spectra of ZnS NPs films prepared and annealed at (300K) and (623K) respectively 
Table (2): (UL) and (BL) of ZnS NPs films prepared at temperature (300k) and (623k)

\begin{tabular}{|c|c|c|c|c|}
\hline Preparing temp. (K) & PL & FWHM (nm) & Intensity & $\lambda(\mathrm{nm})$ \\
\hline \multirow{3}{*}{ As grown $(300 \mathrm{~K})$} & UL & 62 & 29.8 & 396 \\
\hline & BL & 111 & 38.6 & 437 \\
\hline & UL & 77.5 & 16.6 & 397 \\
\hline Annealing temp. (623K) & BL & 118 & 23.2 & 439 \\
\hline
\end{tabular}

\section{Conclusion}

1 - From the absorbance spectra for $\mathrm{ZnS}$ thin films, it is concluded that the maximum absorption peaks shift towards the smaller wavelength with the increase of annealing temperatures. The value of absorption and reflection decreases with the increases of annealing temperatures whereas the transmission increases.

2- The optical energy gap for $\mathrm{ZnS}$ increases with the increase in the annealing temperatures.

3- Increasing number pulses of laser on the samples leads to increased roughness of the surface and increased temperature reduces.

4- ZnS NPs films at $300 \mathrm{~K}^{\circ}$ and the transmittance increased after annealing at $623 \mathrm{~K}$ with increasing number pulses of laser.

5- The grain size increases with the increase of absorption.

6- The surface was uniformly covered and the observed surface properties have a strong effect on the optical properties of thin films such as transition, absorption, and reflection.

7- The absorption coefficient $(\alpha)>10^{4} \mathrm{~cm}^{-1}$ indicates direct transitions.

8- Film annealing leads to improving crystallinity, increasing of the grain size and eliminating some defects from the films.

9- The carrier concentration decreases with the increase of annealing temperatures, and the carrier mobility increases with the increase of annealing temperatures.

\section{References}

1- A. Nitta , K. Tanakab, Y. Maekawab, M. Kusabirakib, and Masao Aozasa, Effects of gas impurities in the sputtering environment on the stoichiometry and crystallinity of $\mathrm{ZnS}: \mathrm{Mn}$ electroluminescent-device active layers, Thin Solid Films. 384(2001), 261-268.

2- S. Yano, R. Schroeder, B. Ullrich, and H. Sakai, Absorption and photocurrent properties of thin $\mathrm{ZnS}$ films formed by pulsed-laser deposition on quartz, Thin Solid Films. 423(2003) 273-276.
3- Q.J. Feng, D.Z. Shen, J.Y. Zhang, H.W. Liang, D.X. Zhao, Y.M. Lua, and X.W. Fan, Highly aligned $\mathrm{ZnS}$ nanorods grown by plasma-assisted metalorganic chemical vapor deposition, J. Crystal Growth. 285(2005) 561-565.

4- S. Wang, X. Fu, G. Xia, J. Wang, J. Shao, and Z. Fan, Structure and optical properties of $\mathrm{ZnS}$ thin films grown by glancing angle deposition, Appl. Surf. Sci., 252 (2006) 8734-8737.

5- N. Fathy, R. Kobayashi, and M. Ichimura, Preparation of $\mathrm{ZnS}$ thin films by the pulsed electrochemical deposition, Mater. Sci. Eng. B. 107(2004) 271-27.

6- P. Roy, J.R. Ota, and S.K. Srivastava, Crystalline $\mathrm{ZnS}$ thin films by chemical bath deposition method and its characterization, Thin Solid Films. 515(2006) 1912-1917.

7- W.L. Davidson, Xray diffraction evidence for $\mathrm{ZnS}$ formation in zinc activated rubber vulcanizates. phys Rev. 74(1948) 116-117.

8- S.Biswas, S.kar, abrication of $\mathrm{ZnS}$ nanoparticles and nanorods with cubic and hexagonal crystal structures : a sample solvothermal approach, Nanotechnology. 19 (2008) 045710.

9- H. wany D. Ahnj Huik, H. Sony, Structural and optical Properties of $\mathrm{ZnS}$ thin films deposited by RF magnetron suputtering, Nanoscale Reslett. (2012) 7-26.

10- D. Hariskos, B. Fuchs, R. Menner, N. Naghavi, Hubertc, D. Lincot, M. powalia, The $\mathrm{Zn}(\mathrm{S}, \mathrm{O}, \mathrm{OH}) / \mathrm{ZnMgo}$ buffer in thin film $\mathrm{Cu}(\mathrm{In}, \mathrm{Ga})$ $(\mathrm{Se}, \mathrm{S})$ 2-based solar cell part 11 : magnetron suppttering of the ZnMgo buffer layer for in - line Co- evaporated $\mathrm{Cu}(\mathrm{In}, \mathrm{Ga}) \mathrm{Se} 2$ solar cells prog photovolt pes" 17 (2009) 479-488.

11- X.S. Fxany, H. Yec, X.S. Peng, Y.H. wang, W. Uyc, L.D. Zhang, Large- scale synthesis of $\mathrm{ZnS}$ nanosheets by the evaporation of $\mathrm{ZnS}$ nanopowders, J cryst Growth. 263 (2004) 263-268.

12- X. YWang, C. Zhuy, H. Fan, M.F Zhang, X. Ibj, H. Zwang, Growth of ZnS Microfans and nanosheets : Controllable morphology and phase, J. cryst Growth. 310 (2008) 2525-2531.

13- A. Ichiboshi, M. Hongo, T. Akamine, T. Dobashi, T. Nakada, Ultrasonic chemical bath depost of $\mathrm{ZnS}(\mathrm{O}, \mathrm{OH})$ buffer layers and its application to 
CIGS thin film solar cells, sol energy mater So: cells. 90 (2006) 3130-3135.

14- A. Inamet, Low magnetic flux noise observed in laser deposited in situ films of yB2 Cu3Oy and implications for high - TC SQUIDS". In: Nature 341 O, pp. (1980) $723-726$

15- W.H. Bloss, F. Plisterer, H.W. Schock, Advances in Solar energy, and Annual review of research \& development. Vol. 4(1988) P-275.

16- C. Suryanarayana, M. GNorton, X-ray diffraction a practical approach, New York: Plenum Press; 1998.
17- M. Ethayaraja, C. Ravikumar, D. Muthukumaran, K. Dutta, R.J. Bandyopadhyaya, CdS-ZnS coreshell nanoparticle formation: Experiment mechanism and simulation, J. Phys. Chem. 111 (2007) 3246-3252. 